OPEN ACCESS

Edited by:

Alvin H. Schmaier,

Case Western Reserve University,

United States

Reviewed by:

Wei Li,

Marshall University, United States

Coen Maas,

University Medical Center Utrecht,

Netherlands

*Correspondence:

Ana Acacia S. Pinheiro

acacia@biof.ufrj.br

Specialty section:

This article was submitted to

Hematology,

a section of the journal

Frontiers in Medicine

Received: 14 December 2018

Accepted: 27 March 2019

Published: 16 April 2019

Citation:

Silva $L S$, Pinheiro $A S$, Teixeira $D E$

Silva-Aguiar RP, Peruchetti DB,

Scharfstein J, Caruso-Neves $C$ and

Pinheiro AAS (2019) Kinins Released

by Erythrocytic Stages of Plasmodium

falciparum Enhance Adhesion of

Infected Erythrocytes to Endothelial Cells and Increase Blood Brain Barrier

Permeability via Activation of

Bradykinin Receptors.

Front. Med. 6:75.

doi: 10.3389/fmed.2019.00075

\section{Kinins Released by Erythrocytic Stages of Plasmodium falciparum Enhance Adhesion of Infected Erythrocytes to Endothelial Cells and Increase Blood Brain Barrier Permeability via Activation of Bradykinin Receptors}

Leandro S. Silva ${ }^{1}$, Alessandro S. Pinheiro ${ }^{1}$, Douglas E. Teixeira ${ }^{1}$, Rodrigo P. Silva-Aguiar ${ }^{1}$, Diogo B. Peruchetti ${ }^{1}$, Julio Scharfstein ${ }^{1}$, Celso Caruso-Neves ${ }^{1,2}$ and Ana Acacia S. Pinheiro ${ }^{1 *}$

${ }^{1}$ Instituto de Biofísica Carlos Chagas Filho, Universidade Federal do Rio de Janeiro, Rio de Janeiro, Brazil, ${ }^{2}$ Instituto Nacional de Ciência e Tecnologia em Medicina Regenerativa, INCT-Regenera, Conselho Nacional de Pesquisa e Desenvolvimento (CNPq), Rio de Janeiro, Brazil

Background: Plasmodium falciparum, the etiologic agent of malaria, is a major cause of infant death in Africa. Although research on the contact system has been revitalized by recent discoveries in the field of thrombosis, limited efforts were done to investigate the role of its proinflammatory arm, the kallikrein kinin system (KKS), in the pathogenesis of neglected parasitic diseases, such as malaria. Owing to the lack of animal models, the dynamics of central nervous system (CNS) pathology caused by the sequestration of erythrocytic stages of $P$. falciparum is not fully understood. Given the precedent that kinins destabilize the blood brain barrier (BBB) in ischemic stroke, here we sought to determine whether Plasmodium falciparum infected erythrocytes (Pf-iRBC) conditioned medium enhances parasite sequestration and impairs BBB integrity via activation of the kallikrein kinin system (KKS).

Methods: Monolayers of human brain endothelial cell line (BMECs) are preincubated with the conditioned medium from Pf-iRBCs or RBCs (controls) in the presence or absence of HOE-140 or DALBK, antagonists of bradykinin receptor B2 (B2R) and bradykinin receptor $B 1(B 1 R)$, respectively. Following washing, the treated monolayers are incubated with erythrocytes, infected or not with $P$. falciparum mature forms, to examine whether the above treatment (i) has impact on the adhesion of $P f$-iRBC to BMEC monolayer, (ii) increases the macromolecular permeability of the tracer BSA-FITC, and (iii) modifies the staining pattern of junctional proteins (ZO- 1 and $\beta$-catenin).

Results: We found that kinins generated in the parasite conditioned medium, acting via bradykinin B2 and/or B1 receptors (i) enhanced Pf-iRBC adhesion to the endothelium monolayer and (ii) impaired the endothelial junctions formed by ZO-1 and $\beta$-catenin, consequently disrupting the integrity of the BBB. 


\begin{abstract}
Conclusions: Our studies raise the possibility that therapeutic targeting of kinin forming enzymes and/or endothelial bradykinin receptors might reduce extent of Pf-iRBC sequestration and help to preserve BBB integrity in cerebral malaria (CM).
\end{abstract}

Keywords: malaria, Plasmodium falciparum, KKS, bradykinin, endothelial barrier

\section{INTRODUCTION}

Still recognized as major cause of death in Africa, severe malaria is a complex multi-system disorder caused by infection with Plasmodium falciparum. Afflicting millions of people per year [219 million cases worldwide in WHO (1)], the major complications of acute infection with $P$. falciparum are cerebral malaria (CM), pulmonary edema, acute renal failure, severe anemia, and/or bleeding (2). Although the number of patients that develop CM is relatively low (incidence of 1,120/100,000 infected children/year in the endemic areas of Africa), the lethality in children under 5 years old is high (3-5).

The human phases of the malaria life cycle include a silent liver stage which produces infective merozoytes that, subsequently, establish the blood phase of the disease, by invading erythrocytes. Within red blood cells, several cycles of asexual reproduction occur resulting in elevated number of parasites and human disease (6).

In Plasmodium falciparum malaria, erythrocytes containing mature parasites are sequestered in the brain vascular bed, consequently causing obstruction of microvessels, reduced blood flow, and cerebral hypoxia (7). Proinflammatory cytokines are thought to aggravate the infection-associated microvasculopathy that characterizes severe disease (8). This observation is followed by the accumulation of activated platelets and leukocytes, including $\mathrm{CD}^{+} \mathrm{T}$ cells, within the brain microvasculature (9). Besides the classical components required for the development of effector $\mathrm{T}$ lymphocytes, there are indications in the literature that the renin angiotensin system (RAS) is involved in this process (10). Accordingly, our group demonstrated that angiotensin II (Ang II) acts as a co-stimulatory molecule during activation and development of effector function of $\mathrm{CD}^{+} \mathrm{T}$ cells in vitro and in vivo, by using Ag-specific transgenic mice lacking the AT1 receptor (11-13). Moreover, we have also demonstrated that cerebral edema as well as the infiltration of $\mathrm{T}$ cells into the brain of infected mice were attenuated by captopril, the inhibitor of angiotensin-converting enzyme (ACE) (14). The dual-role of ACE connects two distinct proteolytic pathways: RAS and the kallikrein kinin system (KKS). Besides to be responsible for the formation of Ang II, ACE is also able to degrade kinins (15). KKS is an inflammatory mechanism that proteolytically generates proinflammatory kinins, such as the proinflammatory bradykinin (BK). In infectious diseases, the unbalance between pro and anti-coagulant responses may influence infectiousassociated vasculopathies (16). In the past years, progress in the understanding of the role of the KKS in the pathogenesis of experimental Chagas disease revealed that BK-induced microvascular leakage translates into mutual benefits to the host/parasite relationship $(17,18)$.
In the malaria field, although the notion that sequestration of infected erythrocytes to the microvascular brain endothelium is required for the development of $\mathrm{CM}$ is well-accepted $(19,20)$, there have been reports that patients infected with Plasmodium vivax develop CNS pathology without obvious signs of parasite sequestration in the brain $(21,22)$. Experimental models of cerebral malaria (ECM) have unveiled a number of common pathogenic features with the human CM. For example, in both cases the infection-associated vasculopathy includes platelet activation, coagulopathy, vascular leakage, edema, microhemorrages, vascular occlusion, and adhesion of activated leukocytes (23-25). Also, it has been proposed that $P f$-iRBC might activate the KKS through contact activation by surface-exposed phophatidylserine (26-29). Relying on falcipains, $P f$-iRBC directly cleaves internalized kininogens, to release proinflammatory kinins, such as BK Bagnaresi et al. (30). More recently, we demonstrated that the short-lived BK is detectable (mass spectrometry) in culture supernatants of $P f$ iRBCs treated with ACE inhibitors (31). Using monolayers of BMECs as a model of $\mathrm{BBB}(32,33)$, here we provide in vitro evidence that $\mathrm{BK}$ accumulating in the supernatant of $P f$-iRBC cultures (i) enhance the adhesion of $P f$-iRBC adhesion to BMECs (ii) impair the integrity of the brain blood barrier.

\section{MATERIALS AND METHODS}

\section{Drugs}

D-sorbitol, HEPES, glucose, sodium bicarbonate, hypoxanthine, bradykinin (Arg-Pro-Pro-Gly-Phe-Ser-Pro-Phe-Arg), B2 receptor (B2R) antagonist, $\mathrm{HOE}-140, \mathrm{~B} 1$ receptor (B1R) antagonist des-Arg ${ }^{9}-\left[\mathrm{Leu}^{8}\right]-\mathrm{BK}$ (DALBK) were purchased from Sigma-Aldrich.

\section{Ethics Statement}

Healthy volunteers were randomly selected for collection of $\mathrm{A}^{+}$ blood samples. All procedures were approved by the Research Ethics Committee of the Hospital Universitário Clementino Fraga Filho from the Federal University of Rio de Janeiro (Permit Number 074/10). All volunteers provided written informed consent for the collection and subsequent use of the samples to maintain parasite cultures.

\section{Parasite Culture}

Plasmodium falciparum from the W2 strain (chloroquine resistant, mefloquine sensitive), were cultured in RPMI 1,640 medium (Invitrogen) supplemented with $50 \mu \mathrm{g} / \mathrm{mL}$ gentamicin (Invitrogen) and $10 \% \mathrm{~A}^{+}$-type human plasma at $5 \% \mathrm{~A}^{+}$hematocrit, obtained from healthy donors, using citrate as anticoagulant agent. Parasite cultures were maintained under a gas-controlled atmosphere $\left(5 \% \mathrm{CO}_{2}, 5 \% \mathrm{O}_{2}\right.$, and $\left.90 \% \mathrm{~N}_{2}\right)$ as 
described by Trager and Jansen (34). Parasitemia was assessed by light microscopy in thin blood smears stained with hematologic staining by analyzing at least 10 random microscopic fields. Parasitemia was calculated as a percentage of the number of infected cells in 100 erythrocytes.

\section{Culture Synchronization and Generation of the Conditioned Medium}

Erythrocytic stages of $P$. falciparum were synchronized by treatment with $5 \%$ D-sorbitol $(10 \mathrm{~min})$. Mature forms of malaria parasites are known to have osmotic fragility and are sensitive to 5\% D-sorbitol (35). After discarding mature forms, the suspension of parasitized erythrocytes, enriched in young trophozoites, was washed and reintroduced in the above described culture medium to allow for schizont formation. Conditioned medium was obtained by incubating schizont cultures (3-5\% parasitemia) for $24 \mathrm{~h}$, a timepoint in which schizonts give rise to young trophozoites. After centrifugation of $P f$-iRBC $(2,500 \mathrm{rpm}$, for $10 \mathrm{~min})$, the supernatant (conditioned medium) was collected and freshly applied to BMEC monolayers. As control, the supernatant of non-infected erythrocytes suspensions, maintained in the same culture conditions of infected erythrocytes, for $24 \mathrm{~h}$, was used.

\section{Brain Microvascular Endothelial Cell (BMEC) Culture}

The brain microvascular endothelial cells (BMEC) are an immortalized cell line that has been previously described and used as a BBB model in studies of the trans-migration of African trypanosomes $(32,33)$. The BMECs were cultured in medium 199 (M199, Sigma Aldrich) supplemented with 10\% heat-inactivated fetal calf serum (Invitrogen, Carlsbad, CA) and antibiotics (Sigma Chem Co; St. Louis, MO) (complete medium), at $37^{\circ} \mathrm{C}$ in a humidified atmosphere containing $5 \% \mathrm{CO}_{2}$.

\section{Adhesion Assay}

To assess $P f$-iRBC adhesion to endothelial cells, BMEC were plated in 24-well culture chambers (Nunc, New York, USA) $\left(5 \times 10^{4}\right.$ cells/well $)$ and cultured for $24 \mathrm{~h}$. After that, BMECs were treated or not overnight with $P f$-iRBC conditioned medium (20\%) or control conditioned medium (normal RBC). Where indicated, the BMEC treatment with conditioned medium was performed in medium supplemented with the B2R or B1R antagonists $\left(10^{-7} \mathrm{M}\right.$ HOE-140 or DALBK). Next, the BMECtreated monolayers were incubated with $P f$-iRBCs $\left(4 \times 10^{5}\right.$ cells/well, 5\% parasitemia) or control RBCs for $1 \mathrm{~h}$. Nonadherent erythrocytes were gently washed away with PBS, and the remaining cells were fixed and stained with hematologic staining (commercial kit from Laborclin, Brazil, BR). The number of adhered erythrocytes per BMEC was determined by direct counting in light microscopy, considering at least 10 random microscopic field. The data are expressed as Adhesion index calculated according to Souza et al. (36): Adhesion Index (AI) $=\{[($ BMEC with bound erythrocytes $) /$ total BMEC number $] \times$ [(erythrocytes bound to BMEC)/total BMEC number] $\} \times 100$.

\section{Permeability Assay}

Permeability was accessed through BSA-FITC transendothelial transport (37). Briefly, BMEC cell line was grown until confluence on Transwell chamber inserts of $6.5 \mathrm{~mm}$ diameter and $8 \mu \mathrm{m}$ pore (Corning Costar). The endothelial monolayer culture in the upper compartment was exposed to RPMI control medium, Pf-iRBC ( $4 \times 10^{5}$ cells), $20 \%$ conditioned medium, $10^{-7}$ bradykinin (BK), $10^{-7} \mathrm{HOE}$ 140. BSA-FITC $(15 \mu \mathrm{g} / \mathrm{mL})$ was simultaneously added to the upper compartment of each Transwell unit. After $14 \mathrm{~h}$ of incubation times, endothelial monolayer permeability of BSA-FITC flux across intact monolayers to the lower compartment was measured through fluorimetry (SpectraMax M2, Molecular Devices) at emission/excitation wavelengths of 495/520 nm.

\section{Immunofluorescence}

The immunofluorescence experiments were carried out as before $(38,39)$. Briefly, BMEC cells were grown in coverslips and treated as described above. After treatment, cells were fixed in paraformaldehyde $4 \%$ for $15 \mathrm{~min}$, followed by membrane permeabilization with PBS-Triton X-100 $0.2 \%$ for $15 \mathrm{~min}$. Cells were blocked with PBS-BSA 5\%, and antibody against ZO-1 (617,300, Invitrogen) and $\beta$-catenin (sc-7963, Santa Cruz Biotechnology) was incubated for $1 \mathrm{~h}$ at room temperature. Anti-rabbit Alexa-Fluor 488 and Anti-mouse Alexa-Fluor 546 (Life Technology) was incubated to detect ZO-1 and $\beta$-catenin, respectively. Nuclei were stained with DAPI. Cells were mounted with anti-fading mounting medium (Vectashield, Vector Laboratories). Images were acquired with a confocal microscope Leica TCS SP8 (Leica) and software LAS X, and the final images were analyzed with Fiji software. Images were acquired at $630 \mathrm{x}$ and the scale bar represents $20 \mu \mathrm{m}$.

\section{HK-Alexa Fluor 488 Uptake}

The HK-Alexa Fluor 488 uptake was measured as described by Bagnaresi et al. (30). Briefly, schizont-enriched P. falciparum cultures were incubated overnight with $70 \mu \mathrm{g} / \mathrm{mL}$ high molecular weight kininogen conjugated to Alexa Fluor 488 (HK-Alexa Fluor $488)$ at $37^{\circ} \mathrm{C}$. The cells were harvested, washed with PBS $1 \mathrm{x}$ and plated in poly-lysine-coated microscope dishes. Parasites nuclei were stained with DAPI. Images were acquired with a confocal microscope Leica TCS SP8 (Leica) and software LAS X, and the final images were analyzed with Fiji software. Images were acquired at $630 \mathrm{x}$ and the scale bar represents $5 \mu \mathrm{m}$.

\section{Statistical Analysis}

The results are expressed as means \pm standard error of at least three independent experiments. GraphPad Prism 7 (version 7.0, GraphPad Software, San Diego California, U. S. A., www. graphpad.com) was used for statistical analysis. Differences between groups were compared by one-way analysis of variance (ANOVA), followed by the Tukey post-test. Significance was determined as $P<0.05$. 


\section{RESULTS}

\section{$P$ falciparum Conditioned Medium Increases Sequestration of Infected Erythrocytes to BMEC Monolayers}

Although it is well-established that iRBCs are recognized and sequestered by the endothelium, the influence of soluble compounds produced during the erythrocytic cycle in this process is still poorly known. Thus, in the first experimental group, we analyzed the influence of $P$. falciparum conditioned medium in the sequestration of $P f$-iRBCs to BMEC monolayers Conditioned medium was originated from a $24 \mathrm{~h}$ schizont culture (3-5\% parasitemia) as described in the Material and methods section. For controls, we obtained supernatants from $24 \mathrm{~h}$ cultures of non-infected erythrocytes. Sub-confluent BMEC cultures were treated with the respective supernatant, overnight, and subsequently exposed to fresh $P f$-iRBCs, for $1 \mathrm{~h}$. We observed that the pre-incubation of BMEC with increasing proportions of the conditioned medium enhanced $P f$ iRBC adhesion at $20 \%$ (Figures $\mathbf{1 A}, \mathbf{B})$. At this concentration,

A

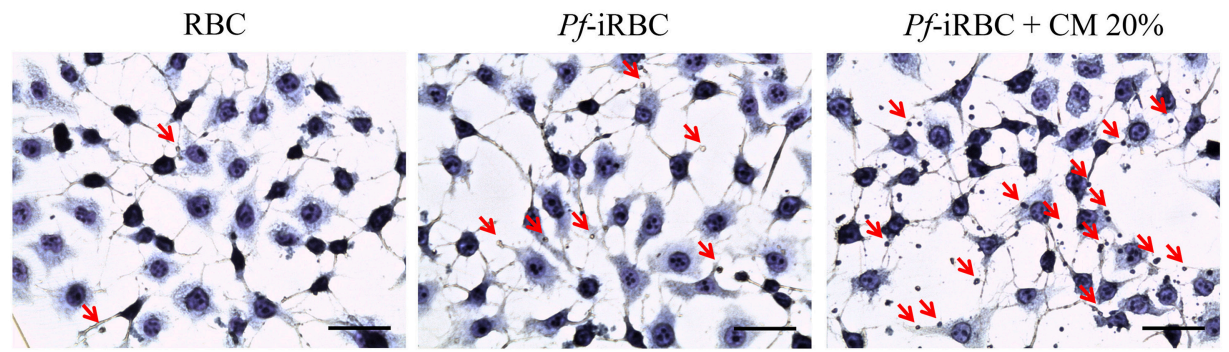

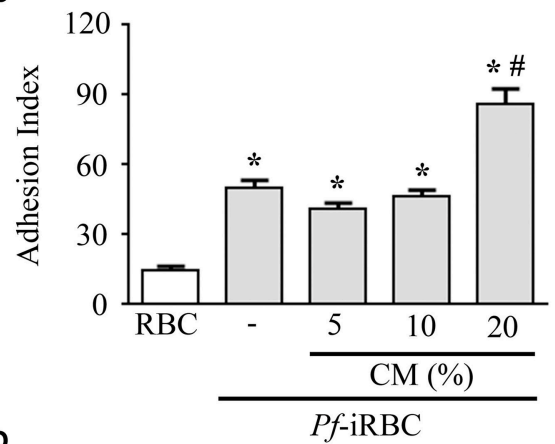

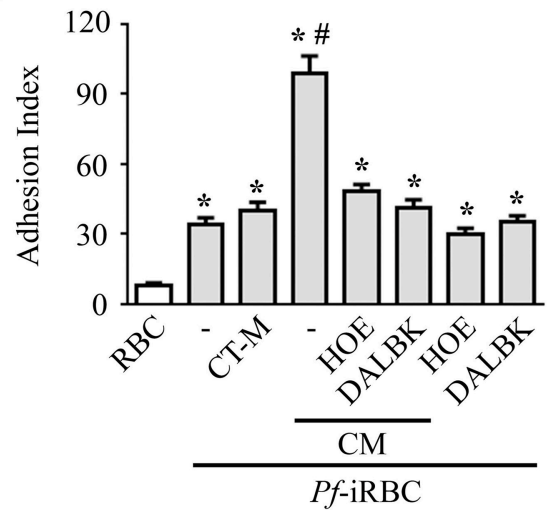

C

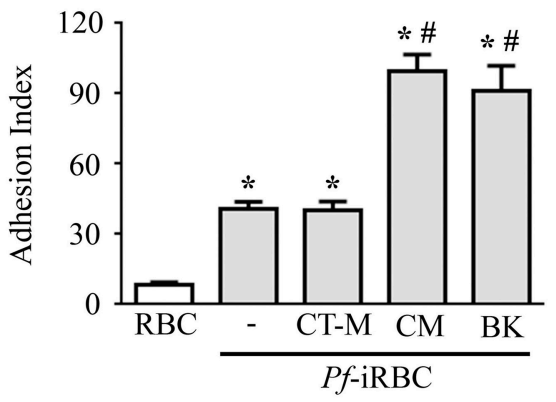

$\mathbf{E}$

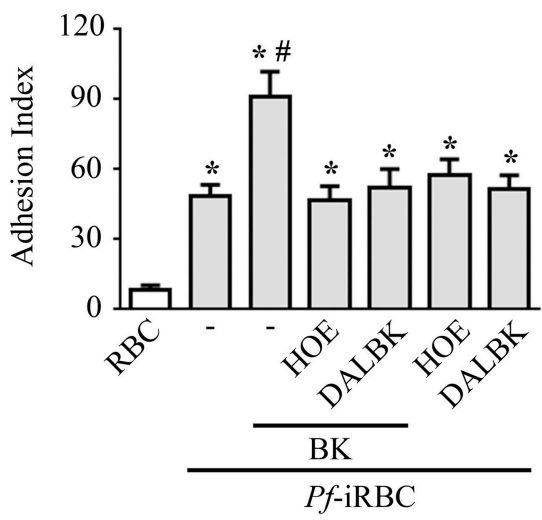

FIGURE 1 | Adhesion of Pf-iRBCs to BMEC monolayers is increased by the supernatant from $P$. falciparum culture. BMEC were cultured in 24 -well plates $\left(4 \times 10^{5}\right.$ cells/well) for $24 \mathrm{~h}$ and incubated with Pf-iRBCs (5 $\times 10^{4}$ erythrocytes/well, $5 \%$ parasitemia). When indicated, BMEC were treated with different compounds before incubation with Pf-iRBCs. The adhesion index was determined by direct counting of adhered erythrocytes per BMEC, as described in the Materials and methods. (A) Representative images of adhesion of $P f$-iRBCs to BMEC monolayers. Arrows indicates adhered $P f$-iRBCs. Scale bar $=50 \mu \mathrm{m}$. (B) Adhesion index was determined in BMEC preincubated or not with increasing concentrations of Pf-iRBC conditioned medium (CM), overnight, before incubation with $P f$-iRBCs. (C) Adhesion index

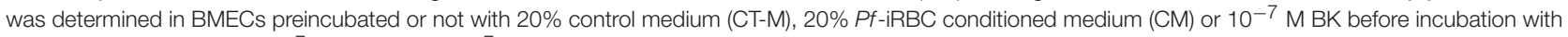
Pf-iRBCs. (D,E) Effect of $10^{-7} \mathrm{M}$ DALBK or $10^{-7} \mathrm{M} \mathrm{HOE}-140$ on the adhesion of Pf-iRBCs to BMEC. BMECs were pretreated with drugs for 30 min prior to overnight incubation with conditioned medium (D) or BK (E). CT-M, control medium, obtained from a suspension of non-infected erythrocytes; CM, $P$. falciparum conditioned medium; RBC, basal adherence of non-infected erythrocytes; $P f$-iRBC, $P$. falciparum-infected erythrocytes. Results are expressed as the mean $\pm \mathrm{SEM}$ from three different experiments. *Statistically significant differences compared with RBCs, \#statistically significant different compared with iRBCs $(P<0.05)$. 
the conditioned medium produced 2-fold increase in $P f$ iRBCs binding to endothelial cells. The stimulatory effect of the conditioned medium was comparable to the effect of addition of $10^{-7} \mathrm{M} \mathrm{BK}$ alone (Figure 1C). To characterize the receptors activated by the parasite conditioned medium, we preincubated the monolayer of BMECs with $10^{-7} \mathrm{M}$ DALBK (B1R antagonist) or HOE-140 (B2R antagonist) before adding the $P f$-iRBC conditioned medium (Figure 1D) or BK (Figure 1E). Notably, both antagonists abolished the subsequent adhesion of $P f$-iRBC to BMECs. Of further interest, none of these GPCR blockers changed the basal levels of adhesion of infected erythrocytes. These results suggest that the adhesion of erythrocytic stages of $P$. falciparum to BMECs was enhanced by kinins generated in the $\mathrm{Pf}$ iRBC conditioned medium (30). Accordingly, when infected cultures enriched with mature forms of the parasite were incubated with $70 \mu \mathrm{g} / \mathrm{mL}$ HK-Alexa Fluor 488, we observed the fluorescent substrate inside infected cells, revealed by immunofluorescence, but not in non-infected erythrocytes (Figure 2).

\section{P. falciparum Conditioned Medium Directly Induces Permeability of the BMEC Monolayer}

Using fluorimetry and album-FITC as a macromolecular tracer, we sought to measure the permeability of a confluent BMEC monolayer prepared in a transwell system. The permeability measurements were made after incubating the BMEC monolayer overnight with $20 \%$ P. falciparum conditioned medium vs. $20 \%$ control medium (CT-M) obtained from $24 \mathrm{~h}$ culture of noninfected erythrocytes. Our results showed that the conditioned medium induced a 10 -fold increase in BMEC permeability over the effect of the control (Figure 3). Of note, the intensity of the permeability response induced by $P f$-iRBC conditioned medium was equivalent to responses induced by $10^{-7} \mathrm{M} \mathrm{BK}$ alone (Figure 3). Importantly, addition of the B2R antagonist HOE-140 $\left(10^{-7} \mathrm{M}\right)$ stabilized the barrier function in BMECs incubated either with BK or parasite-conditioned medium. As observed in the results obtained in the endothelial cell adhesion assay, we found that the B1R blocker DALBK $\left(10^{-7}\right.$ M) partially protected the barrier from the permeabilityinducing signals generated in the parasite-conditioned medium or BK (Figure 3). Hence, these pharmacological studies linked

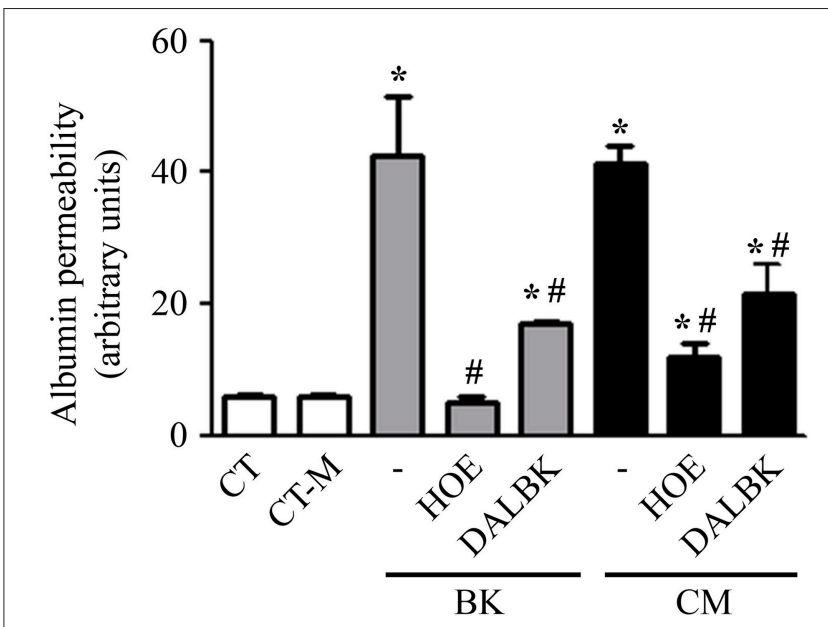

FIGURE 3 | $P$. falciparum conditioned medium induces endothelial barrier disruption in a BKR-dependent manner. Confluent BMEC monolayers, cultivated in the upper chamber of a transwell system, were pretreated or not with $10^{-7} \mathrm{M} \mathrm{HOE} 140$ or $10^{-7} \mathrm{M} \mathrm{DALBK}$, for $30 \mathrm{~min}$, before overnight incubation with $20 \%$ conditioned medium or $10^{-7} \mathrm{M} \mathrm{BK}$. The next day, cells were washed and incubated with $15 \mu \mathrm{g} / \mathrm{mL}$ BSA-FITC, for $15 \mathrm{~min}$. The supernatant from the lower chamber was collected for fluorimetry analysis. Bar graph representing the amount of fluorescence detected in the lower chamber for each experimental setup. Albumin permeability was expressed in arbitrary units as the mean \pm SE of at least three independent experiments. Statistical significance compared with *control; \#BK or conditioned medium $(P<0.05)$.

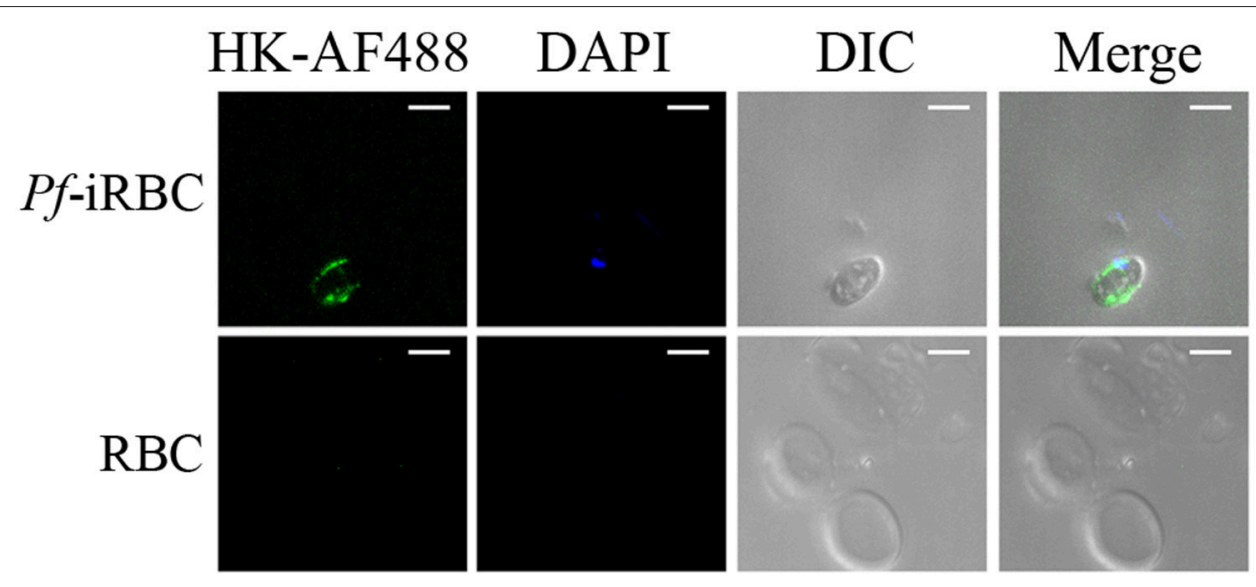

FIGURE 2 | P. falciparum infected erythrocytes uptake high molecular weight kininogen from the extracellular medium. Schizont-enriched $P$. falciparum cultures were incubated overnight with $70 \mu \mathrm{g} / \mathrm{mL}$ high molecular weight kininogen conjugated to fluorescein isothiocyanate (HK-AF488) at $37^{\circ} \mathrm{C}$. The cells were harvested, plated in poly-lysine-coated microscope dishes, and analyzed by fluorescence microscopy. Images were analyzed using green (internalized HK-AF488) and blue (parasite nucleus) filters or DIC (erythrocytes). RBC, non-infected erythrocytes; Pf-iRBC, infected erythrocytes. Scale bar $=5 \mu \mathrm{m}$. 

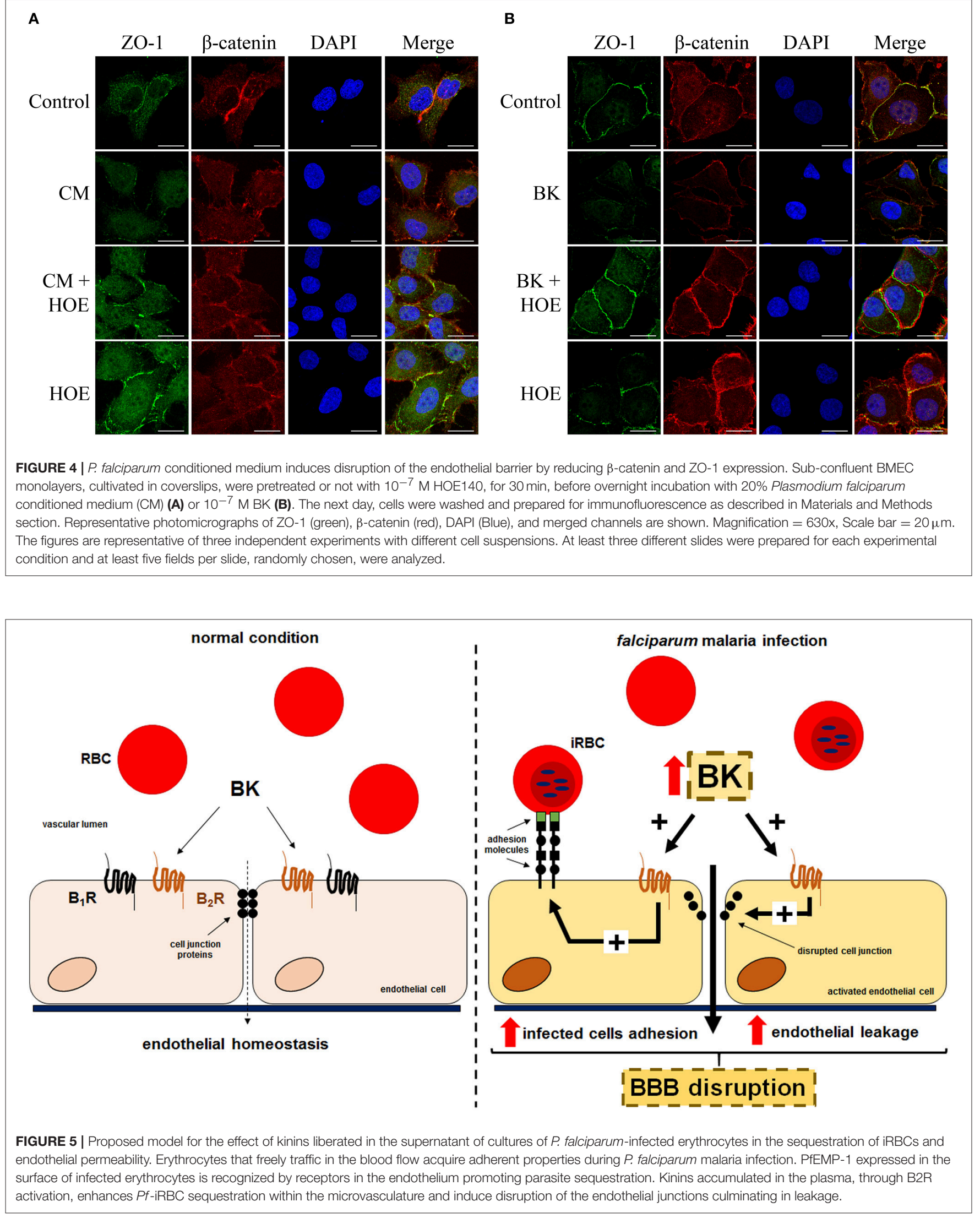
the increased permeability response induced by the $P f$-iRBC conditioned medium to BMEC activation via the kinin/ BKRs pathways.

\section{Kinins/B2R Axis Induces Permeability by Changing Morphological Distribution of Proteins From the Intercellular Junction}

To further investigate the impact of the conditioned medium in BMEC permeability, we examined the morphological distribution of the components involved in the formation of interendothelial junction, e.g., ZO-1 and $\beta$-catenin. Typically, these proteins clearly co-localize in the periphery of the cell maintaining the integrity of the endothelial monolayer. However, in the presence of the parasite-conditioned medium (but not erythrocytes control medium) ZO-1 and $\beta$-catenin staining pattern was not clearly visualized. Consistent with the opening of intercellular gaps, these results suggested that the barrier function of the BMECs was disrupted (Figure 4A). Importantly, the steady-state localization of ZO-1 and $\beta$-catenin were restored by $10^{-7}$ M HOE-140, hence reinforcing the conclusion that kinins, acting via $\mathrm{B} 2 \mathrm{R}$, are responsible for the permeabilityinducing properties of the $P f$-iRBC conditioned medium (Figure 4A). In parallel, the direct effect of $10^{-7} \mathrm{M} \mathrm{BK}$ on the structural assembly of the intercellular junction was evaluated. BK, through $\mathrm{B} 2 \mathrm{R}$ activation, reproduced similar results compared with the addition of $P f$-iRBC supernatant (Figure 4B).

Collectively, our results suggest that kinins generated during cultivation of $P$. falciparum erythrocytic stages might contribute to the pathogenesis of $\mathrm{CM}$ via two distinct, but not mutually exclusive activation pathways. The released $B K$ (i) might favor $P f$-iRBC sequestration within the microvasculature and (ii) disrupt the integrity of the endothelial junctions, inducing interstitial edema (Figure 5). Both mechanisms seem to be dependent on B2R activation.

\section{DISCUSSION}

Although sequestration of erythrocytic stages of $P$. falciparum is thought to be a crucial event in the pathogenesis of CM (7), the hemostatic derangements are thought to be aggravated by formation of microthrombi, local or systemic production of proinflammatory cytokines. Despite progress in the molecular characterization of the parasite factors that promote adhesive interactions of $P f$-iRBC with the endothelium $(40,41)$, the lack of experimental models to investigate the dynamics of infection by human species of Plasmodium has limited progress in this field. In vitro experiments using mature forms (schizonts) of parasitized erythrocytes showed upregulated endothelial expression of tissue factor, a trigger of fibrin-formation via the extrinsic pathway of coagulation (27). Multiple mechanisms may promote intravascular activation of the contact system (intrinsic pathway) following the sequestration of $P f$-iRBC in the cerebral microvessels. For example, it is well-established that formation of microthrombi is potentiated by fibrin as a result of contact system activation by negatively charged platforms (26-29). Along similar lines, DNA associated to neutrophil extracellular traps (NETs) was also identified as a trigger of Factor XII, the serine protease that generates plasma kallikrein, the main BK-forming serine protease in the blood $(15,42)$.

Early in vitro studies performed by our group revealed that parasitized erythrocytes might modulate immunity and vascular homeostasis via activation of the reninangiotensin system (RAS) (11-13). Given awareness that the hypertensive ACE efficiently degrades kinins, follow up studies performed with ACE inhibitors identified the presence of this nanopeptide in the parasite conditioned medium (31). Interestingly, in this context, independent studies by Bagnaresi et al. (30) demonstrated that high molecular weight kininogen is internalized and processed by E-64sensitive kinin releasing cysteine proteases in different species of Plasmodium.

Although we did not investigate the mechanisms by which $P$. falciparum might generate kinins in the conditioned medium, the levels of this short-lived nanopeptide were sufficiently high to destabilize endothelial junctions and promote enhanced diffusion of the albumin-FITC tracer through the BMECs. B1R antagonist (DALBK) inhibited the adhesion of $P f$-iRBCs to the endothelial cells as efficiently as B2R antagonist (HOE-140). Also, both antagonists efficiently rescued the barrier function of BMECs, along with the restoration of the peripheral staining of $\beta$-catenin and $\mathrm{ZO}$ 1 in the BMECs. In summary, our results suggest that kinins generated by erythrocytic stages of $P$. falciparum might disrupt $\mathrm{BBB}$ integrity following parasite sequestration in microvessels.

\section{ETHICS STATEMENT}

Healthy volunteers were randomly selected for collection of $\mathrm{A}+$ blood samples. All procedures were approved by the Research Ethics Committee of the Hospital Universitário Clementino Fraga Filho from the Federal University of Rio de Janeiro (Permit Number 074/10). All volunteers provided written informed consent for the collection and subsequent use of the samples to maintain parasite cultures.

\section{AUTHOR CONTRIBUTIONS}

LS performed all experiments, collected, organized and analyzed all data. ASP and DT performed all cell cultures, parasite synchronization, and adhesion experiments. RS-A performed immunofluorescence experiments and helped with data organization. DP helped with drafting and revision of the manuscript. JS and CC-N helped with data interpretation, drafting, and revision of the manuscript; 
AASP conceived and designed the work, drafted, and revised the manuscript.

\section{FUNDING}

This work was supported by grants from the following Brazilian agencies: Conselho Nacional de Desenvolvimento Científico e Tecnológico (www.cnpq.br) 304682/2015-2 (AASP); 303793/2015-5 (CC-N); and Fundação Carlos Chagas Filho de Amparo à Pesquisa do Estado do Rio de

\section{REFERENCES}

1. World Health Organization. WHO World Malaria Report. Geneva: World Health Organization (2017).

2. Trampuz A, Jereb M, Muzlovic I, Prabhu RM. Clinical review: severe malaria. Crit Care. (2003) 7:315-23. doi: 10.1186/cc2183

3. Idro R, Marsh K, John CC, Newton CR. Cerebral malaria: mechanisms of brain injury and strategies for improved neurocognitive outcome. Pediatr Res. (2010) 68:267-74. doi: 10.1203/PDR.0b013e3181eee738

4. Murray CJ, Rosenfeld LC, Lim SS, Andrews KG, Foreman KJ, Haring D, et al. Global malaria mortality between 1980 and 2010: a systematic analysis. Lancet. (2012) 379:413-31. doi: 10.1016/S0140-6736(12)60034-8

5. Murray CJ, Ortblad KF, Guinovart C, Lim SS, Wolock TM, Roberts $\mathrm{DA}$, et al. Global, regional, and national incidence and mortality for HIV, tuberculosis, and malaria during 1990-2013: a systematic analysis for the Global Burden of Disease Study 2013. Lancet. (2014) 384:1005-70. doi: 10.1016/S0140-6736(14)60844-8

6. Ashley EA, Pyae Phyo A, Woodrow CJ. Malaria. Lancet. (2018) 391:1608-21. doi: 10.1016/S0140-6736(18)30324-6

7. O'Sullivan JM, O’Donnell JS. Platelets in malaria pathogenesis. Blood. (2018) 132:1222-4. doi: 10.1182/blood-2018-08-865618

8. McMorran BJ, Wieczorski L, Drysdale KE, Chan JA, Huang HM, Smith C, et al. Platelet factor 4 and Duffy antigen required for platelet killing of Plasmodium falciparum. Science. (2012) 338:1348-51. doi: $10.1126 /$ science. 1228892

9. Ghazanfari N, Mueller SN, Heath WR. Cerebral malaria in mouse and man. Front Immunol. (2018) 9:2016. doi: 10.3389/fimmu.2018.02016

10. Hoch NE, Guzik TJ, Chen W, Deans T, Maalouf SA, Gratze P, et al. Regulation of T-cell function by endogenously produced angiotensin II. Am J Physiol Regul Integr Comp Physiol. (2009) 296:R208-216. doi: 10.1152/ajpregu.90521.2008

11. Silva-Filho JL, Souza MC, Henriques MG, Morrot A, Savino W, Nunes MP, et al. AT1 receptor-mediated angiotensin II activation and chemotaxis of T lymphocytes. Mol Immunol. (2011) 48:1835-43. doi: 10.1016/j.molimm.2011.05.008

12. Silva-Filho JL, Caruso-Neves C, Pinheiro AAS. Angiotensin II type-1 receptor (AT1R) regulates expansion, differentiation, and functional capacity of antigen-specific CD8+ T cells. Sci Rep. (2016) 6:35997. doi: 10.1038/srep 35997

13. Silva-Filho JL, Caruso-Neves C, Pinheiro AAS. Targeting angiotensin II type1 receptor (AT1R) inhibits the harmful phenotype of plasmodium-specific CD8+ T cells during blood-stage malaria. Front Cell Infect Microbiol. (2017) 7:42. doi: $10.3389 /$ fcimb. 2017.00042

14. Silva-Filho JL, Souza MC, Ferreira-Dasilva CT, Silva LS, Costa MF, Padua TA, et al. Angiotensin II is a new component involved in splenic T lymphocyte responses during Plasmodium berghei ANKA infection. PLoS ONE. (2013) 8:e62999. doi: 10.1371/journal.pone.0062999

15. Schmaier AH. The contact activation and kallikrein/kinin systems: pathophysiologic and physiologic activities. J Thromb Haemost. (2016) 14:28-39. doi: 10.1111/jth.13194

16. Oehmcke $\mathrm{S}$, Herwald $\mathrm{H}$. Contact system activation in severe infectious diseases. J Mol Med. (2010) 88:121-6. doi: 10.1007/s00109-009-0564-y
Janeiro-FAPERJ (www.faperj.br): E-26/202.950/2016 (AASP); E-26/202.833/2017 (CC-N).

\section{ACKNOWLEDGMENTS}

The authors thank Dr. Guacyara da Motta from Federal University of São Paulo to gently provide the HK-Alexa Fluor 488 for the HK-Alexa Fluor 488 uptake assay and Mr. Mario Luiz da Silva Bandeira (Faperj fellowships) for the technical support.

17. Scharfstein J, Ramos PIP, Barral-Netto M. G protein-coupled kinin receptors and immunity against pathogens. Adv Immunol. (2017) 136:29-84. doi: 10.1016/bs.ai.2017.05.007

18. Scharfstein J. Subverting bradykinin-evoked inflammation by co-opting the contact system: lessons from survival strategies of Trypanosoma cruzi. Curr Opin Hematol. (2018) 25:347-57. doi: 10.1097/MOH.0000000000000444

19. Milner DA Jr. Rethinking cerebral malaria pathology. Curr Opin Infect Dis. (2010) 23:456-63. doi: 10.1097/QCO.0b013e32833c3dbe

20. White NJ, Turner GDH, Medana IM, Dondorp AM, Day NPJ. The murine cerebral malaria phenomenon. Trends Parasitol. (2010) 26:11-5. doi: 10.1016/j.pt.2009.10.007

21. Silamut K, Phu NH, Whitty C, Turner GD, Louwrier K, Mai NT, et al. A quantitative analysis of the microvascular sequestration of malaria parasites in the human brain. Am J Pathol. (1999) 155:395-410. doi: 10.1016/S0002-9440(10)65136-X

22. Barber BE, William T, Grigg MJ, Parameswaran U, Piera KA, Price RN, et al. Parasite biomass-related inflammation, endothelial activation, microvascular dysfunction and disease severity in vivax malaria. PLoS Pathog. (2015) 11:e1004558. doi: 10.1371/journal.ppat.1004558

23. Desruisseaux MS, Machado FS, Weiss LM, Tanowitz HB, Golightly LM. Cerebral malaria: a vasculopathy. Am J Pathol. (2010) 176:1075-8. doi: 10.2353/ajpath.2010.091090

24. Cabrales P, Zanini GM, Meays D, Frangos JA, Carvalho LJ. Murine cerebral malaria is associated with a vasospasm-like microcirculatory dysfunction, and survival upon rescue treatment is markedly increased by nimodipine. Am J Pathol. (2010) 176:1306-15. doi: 10.2353/ajpath.2010.090691

25. Nacer A, Movila A, Baer K, Mikolajczak SA, Kappe SH, Frevert U. Neuroimmunological blood brain barrier opening in experimental cerebral malaria. PLoS Pathog. (2012) 8:e1002982. doi: 10.1371/journal.ppat.1002982

26. Eda S, Sherman IW. Cytoadherence of malaria-infected red blood cells involves exposure of phosphatidylserine. Cell Physiol Biochem. (2002) 12:37384. doi: $10.1159 / 000067908$

27. Francischetti IM, Seydel KB, Monteiro RQ. Blood coagulation, inflammation, and malaria. Microcirculation. (2008) 15:81-107. doi: 10.1080/10739680701451516

28. Burnier L, Fontana P, Kwak BR, Angelillo-Scherrer A. Cell-derived microparticles in haemostasis and vascular medicine. Thromb Haemost. (2009) 101:439-51. doi: 10.1160/TH08-08-0521

29. Lang PA, Kasinathan RS, Brand VB, Duranton C, Lang C, Koka S, et al. Accelerated clearance of Plasmodium-infected erythrocytes in sickle cell trait and annexin-A7 deficiency. Cell Physiol Biochem. (2009) 24:415-28. doi: 10.1159/000257529

30. Bagnaresi P, Barros NM, Assis DM, Melo PM, Fonseca RG, Juliano MA, et al. Intracellular proteolysis of kininogen by malaria parasites promotes release of active kinins. Malar J. (2012) 11:156. doi: 10.1186/1475-2875-11-156

31. Silva LS, Peruchetti DB, Silva CTF, Ferreira-DaSilva AT, Perales J, Caruso-Neves C, et al. Interaction between bradykinin B2 and Ang-(1-7) Mas receptors regulates erythrocyte invasion by Plasmodium falciparum. Biochim Biophys Acta. (2016) 1860:2438-44. doi: 10.1016/j.bbagen.20 16.07.011

32. Grab DJ, Garcia-Garcia JC, Nikolskaia OV, Kim YV, Brown A, Pardo CA, et al. Protease activated receptor signaling is required for African trypanosome 
traversal of human brain microvascular endothelial cells. PLoS Negl Trop Dis. (2009) 3:e479. doi: 10.1371/journal.pntd.0000479

33. Nikolskaia OV, Lima AP, Kim YV, Lonsdale-Eccles JD, Fukuma T, et al. Bloodbrain barrier traversal by African trypanosomes requires calcium signaling induced by parasite cysteine protease. J Clin Invest. (2006) 116:2739-47. doi: 10.1172/JCI27798.

34. Trager W, Jensen JB. Human malaria parasites in continuous culture. Science. (1976) 193:673-5. doi: 10.1126/science.781840

35. Lambros C, Vanderberg JP. Synchronization of Plasmodium falciparum erythrocytic stages in culture. J Parasitol. (1979) 65:418-20. doi: $10.2307 / 3280287$

36. Souza MC, Paixão FH, Ferraris FK, Ribeiro I, Henriques Md. Artesunate exerts a direct effect on endothelial cell activation and $\mathrm{nf}-\kappa \mathrm{b}$ translocation in a mechanism independent of plasmodium killing. Malar Res Treat. (2012) 2012:679090. doi: 10.1155/2012/679090

37. Khoy K, Nguyen MVC, Masson D, Bardy B, Drouet C, Paclet MH. Transfusion-related acute lung injury: critical neutrophil activation by anti-HLA-A2 antibodies for endothelial permeability. Transfusion. (2017) 57:1699-708. doi: 10.1111/trf.14134

38. Peruchetti DB, Silva-Aguiar RP, Siqueira GM, Dias WB, Caruso-Neves C. High glucose reduces megalin-mediated albumin endocytosis in renal proximal tubule cells through protein kinase B O-GlcNAcylation. J Biol Chem. (2018) 293:11388-400. doi: 10.1074/jbc.RA117.001337

39. Silva-Aguiar RP, Bezerra NCF, Lucena MC, Sirtoli GM, Sudo RT, Zapata-Sudo G, et al. O-GlcNAcylation reduces proximal tubule proteinreabsorption and promotes proteinuria in spontaneously hypertensive rats. J Biol Chem. (2018) 293:12749-58. doi: 10.1074/jbc.RA118.001746

40. Gillrie MR, Ho M. Dynamic interactions of Plasmodium spp. with vascular endothelium. Tissue Barriers. (2017) 5:e1268667. doi: $10.1080 / 21688370.2016 .1268667$

41. Helms G, Dasanna AK, Schwarz US, Lanzer M. Modeling cytoadhesion of Plasmodium falciparum-infected erythrocytes and leukocytes-common principles and distinctive features. FEBS Lett. (2016) 590:1955-71. doi: 10.1002/1873-3468.12142

42. Schmaier AH. Plasma prekallikrein: its role in hereditary angioedema and health and disease. Front Med. (2018) 5:3. doi: 10.3389/fmed.2 018.00003

Conflict of Interest Statement: The authors declare that the research was conducted in the absence of any commercial or financial relationships that could be construed as a potential conflict of interest.

Copyright (๑ 2019 Silva, Pinheiro, Teixeira, Silva-Aguiar, Peruchetti, Scharfstein, Caruso-Neves and Pinheiro. This is an open-access article distributed under the terms of the Creative Commons Attribution License (CC BY). The use, distribution or reproduction in other forums is permitted, provided the original author(s) and the copyright owner(s) are credited and that the original publication in this journal is cited, in accordance with accepted academic practice. No use, distribution or reproduction is permitted which does not comply with these terms. 